\title{
Coalitional Strategic Behaviour in Collective Decision Making
}

\author{
Grzegorz Lisowski ${ }^{1}$ \\ ${ }^{1}$ Department of Computer Science, University of Warwick, United Kingdom \\ grzegorz.lisowski@warwick.ac.uk
}

\begin{abstract}
In my $\mathrm{PhD}$ project I study the algorithmic aspects of strategic behaviour in collective decision making, with the special focus on voting mechanisms. I investigate two manners of manipulation: (1) strategic selection of candidates from groups of potential representatives and (2) influence on voters located in a social network.
\end{abstract}

\section{Introduction}

The research I conduct is situated in the field of collective decision making. In particular, I investigate the ways in which agents and groups of them can manipulate the outcome of the procedure, using the influence they have on their peers. I plan to devote my thesis to two topics in collective decision making. In the first, I plan to investigate the behaviour of groups of candidates in the elections, trying to ensure that their representative is selected. In the second, I envisage to study the strategic influence of voters in social networks.

The first portion of research I plan as part of my PhD thesis is the development of the line proposed by Faliszewski et al. (2016) who investigate the strategic behaviour of coalitions of politicians (parties) striving to achieve the optimal result as a group. I study how political parties might select their candidates in order to optimize their performance in the elections. A natural way of exploring this direction is to model elections as games in which the utility of a particular party depends on whether it's representative is selected. I study classical game theoretic concepts, such as Nash equilibria, in the considered context. Interesting questions regarding such frameworks involve the characterisation of games which admit the existence pure Nash equilibria, or how hard is it to find an equilibrium state in the election game if it exists? Furthermore, a problem which I would like to investigate is how the strategic behaviour of parties affects the satisfaction of voters. A natural method of investigating this problem is the study of the price of anarchy in the considered mechanisms.

The second line of proposed research is related to the social choice on social network. Suppose that a voter is in a

Copyright (C) 2020, Association for the Advancement of Artificial Intelligence (www.aaai.org). All rights reserved. situation in which voting truthfully is the best strategy given the opinions of other voters. This does not mean that she cannot improve the outcome of the elections by influencing her peers in a strategic manner. In my $\mathrm{PhD}$ thesis I will study how agents can misrepresent their preferences over candidates they communicate to other agents in order to improve the outcome of the elections for themselves. I will study the voting mechanisms in which manipulation by influencing peers is computationally difficult. Further, given a voting method, it would be interesting to identify classes of networks for which manipulation is not possible.

\section{Progress to Date}

In this section I will briefly describe the main contribution of the projects I have completed so far during my PhD studies.

\section{Coalitional Knockout Tournaments}

In this paper, co-authored with Ramanujan Sridharan and Paolo Turrini, I investigate the game-theoretic aspects of knockout tournaments in which teams of players select representatives to compete. We are aiming at publishing the results of this project, which is a part of the first line of research of my thesis, in the Journal of Autonomous Agents and Multi-Agent Systems (JAAMAS).

Knockout tournaments are competitions in which a single winner is selected based on pairwise comparisons between players. More precisely, players are assigned to leafs of a binary tree following a seeding function. They subsequently compete in pairs and the winner advances to the next stage. The competition goes on until only one player remains in the tournament. They have been applied not only in the context of sports. They have been studied in computational social choice, as they constitute a natural voting mechanism (see, e.g. (Williams and Moulin 2016)), which links the discussed project to the collective decision making setting.

In the paper we considered a natural extension of knockout tournaments in which coalitions select their representatives to participate in the competition. We further take into account two types of competitions: in the first teams select one representative each for the entire tournament. In the second, teams are allowed to choose a player in every round. Moreover, we consider two types of utilities: in the 
first, teams are only concerned with winning the competition (win/lose scenario). In the second, they strive to advance to the highest round possible (beyond win/lose scenario).

We have provided an analysis of the algorithmic properties of game-theoretic solution concepts in the considered setting, focusing on Nash equilibria (NE) and dominant strategy equilibria (DSE). We have studied the complexity of finding strategy profiles satisfying the conditions of the solution concepts. We have found that finding DSE is polynomial in all considered settings. Further, finding a NE is possible in polynomial time in the beyond win/lose, dynamic scenario and quasi-polynomial time in all other considered possibilities.

\section{Party Nominees in Hotelling-Downs Model}

In this paper, also co-authored with Ramanujan Sridharan and Paolo Turrini, we study the problem of strategic selection of party candidates given the distribution of voters in the political spectrum. This research is directly related to the first theme of my thesis.

We study the variation of the Hotelling-Downs model (initially introduced by Hotelling (1929)), which is a classical framework for the analysis of strategic selection of policies aimed at maximizing the number of attracted voters. There, people are located in a two-dimensional space corresponding to their political views. Subsequently, parties select their positions on the spectrum, after which every voter selects the option which is closest to them. To account for the nature of selecting nominees, we assume that parties' choices are limited to a finite number of points on the spectrum, corresponding to the views of the politicians.

We investigated the algorithmic aspects of game-theoretic solution concepts in the described context, focusing on pure Nash equilibria (NE) and dominant strategy equilibria (DSE). We have found that while checking if a DSE exists is tractable, checking if there is a NE in a given game is NPcomplete. However, when the number of parties is limited to 2 , the considered problems are solvable in linear time of the size of the input.

\section{Computational Complexity in Opinion Diffusion}

In this paper, co-authored with Dmitry Chistikov, Mike Paterson and Paolo Turrini, I investigate the algorithmic properties of opinion diffusion protocols. More precisely, I study the computational complexity of checking if the spread of opinion terminates given a social network. This paper has been accepted to AAAI '20 (Chistikov et al. Forthcoming). This project is a part of the second theme of my thesis.

Opinion diffusion protocols allow for a study of how opinions spread in social network, modelled as a directed graph over a set of agents, following specified rules (see, e.g, (Axelrod 1997; Grandi, Lorini, and Perrussel 2015)). Among numerous protocols, threshold models (Granovetter 1978) are perhaps the best known. There, agents change their opinion if the specified quota of their influencers disagrees with them. One of the main issues associated with them is that they do not guarantee the termination of the diffusion process.
In the paper we established the complexity of establishing if, given a social network, there exists an initial distribution of opinions such that the protocol does not converge. We have focused on a special case of threshold models with the uniform majority quota. We have found that even though in many special cases the convergence of the protocol is guaranteed, it is PSPACE-complete in the general case.

\section{Further Research}

In the rest of my $\mathrm{PhD}$ studies I plan to expand on the directions started in the described projects. At the moment, I am working on the theoretical aspects of majority illusion, a surprising property of social networks initially observed by Lerman, Yan, and Wu (2016). Given a social network with a binary colouring (corresponding, for instance, to agents' stances with respect to some issue), an illusion occurs if for every vertex, the majority of it's neighbourhood is coloured differently than the majority of vertices in the entire network. This property is closely related to the influence that communication has on voting: assuming that agents change their opinion if the majority of their influencers disagrees with them, we get that the opinion of the minority becomes a consensus. I am investigating the properties of networks which admit such a colouring. In particular, I study the computational complexity of checking if a given network admits an illusion.

In future I will expand on the results of the project on party nominees in Hotelling-Downs model. In particular, I intend to study how a number parties might form coalitions to achieve a better outcome than any of them could on their own.

\section{References}

Axelrod, R. M. 1997. The Dissemination of Culture: a Model with Local Convergence and Global Polarization. The Journal of Conflict Resolution 203-226.

Chistikov, D.; Lisowski, G.; Paterson, M.; and Turrini, P. Forthcoming. Convergence of opinion diffusion is pspacecomplete. Proceedings of the Thirty-Fourth AAAI Conference on Artificial Intelligence.

Faliszewski, P.; Gourvès, L.; Lang, J.; Lesca, J.; and Monnot, J. 2016. How Hard is it for a Party to Nominate an Election Winner? In International Joint Conference on Artificial Intelligence, (IJCAI).

Grandi, U.; Lorini, E.; and Perrussel, L. 2015. Propositional Opinion Diffusion. In International Conference on Autonomous Agents \& Multiagent Systems (AAMAS).

Granovetter, M. 1978. Threshold Models of Collective Behavior. American Journal of Sociology 83(6):1420-1443.

Hotelling, H. 1929. Stability in competition. The Economic Journal 39(153):41-57.

Lerman, K.; Yan, X.; and Wu, X.-Z. 2016. The "majority illusion" in social networks. PloS one 11(2).

Williams, V. V., and Moulin, H. 2016. Knockout Tournaments. In Brandt, F.; Conitzer, V.; Endriss, U.; Lang, J.; and Procaccia, A. D., eds., Handbook of Computational Social Choice. Cambridge University Press. 453-474. 